\title{
Stable epidermal electronic device with strain isolation induced by in situ Joule heating
}

\author{
Zihao Wang ${ }^{1,2}$, Qifeng Lu', Yizhang Xia ${ }^{3}$, Simin Feng ${ }^{1}$, Yixiang Shi', Shuqi Wang ${ }^{1}$, Xianqing Yang ${ }^{1}$, Yangyong Zhao ${ }^{1}$, \\ Fuqin Sun', Tie Li ${ }^{1}$ and Ting Zhang ${ }^{1 凶}$
}

\begin{abstract}
Epidermal electronics play increasingly important roles in human-machine interfaces. However, their efficient fabrication while maintaining device stability and reliability remains an unresolved challenge. Here, a facile in situ Joule heating method is proposed for fabricating stable epidermal electronics on a polyvinyl alcohol (PVA) substrate. Benefitting from the precise control of heating locations, the crystallization and enhanced rigidity of PVA are restricted to desired areas, leading to strain isolation of the active regions. As a result, the electronic device can be conformably attached to skin while showing negligible degradation in device performance during deformation. Based on this method, a flexible surface electromyography (sEMG) sensor with outstanding stability and highly comfortable wearability is demonstrated, showing high accuracy (91.83\%) for human hand gesture recognition. These results imply that the fabrication method proposed in this research is a facile and reliable approach for the fabrication of epidermal electronics.
\end{abstract}

\section{Introduction}

Flexible electronics are considered the next revolution in the electronics industry due to their potential applications in areas unreachable with rigid devices ${ }^{1-6}$. As a vital part of flexible electronics, epidermal electronics can be essentially applied in the area of health monitoring and humanmachine interfaces (HMIs) ${ }^{7,8}$. To achieve highly comfortable wearability and outstanding device performance, a stretchable and ultraflexible epidermal electronic device is, by tuning the material modulus and device structures, is desirable to achieve conformable contact with human $\operatorname{skin}^{4,9}$. Generally, there are two strategies to fabricate epidermal electronics. The first method is to fill polymer composites with conductive materials, such as carbon nanotubes, graphene, and metal nanowires ${ }^{10-16}$, while the other method uses metals with designed structures,

\footnotetext{
Correspondence: Ting Zhang (tzhang2009@sinano.ac.cn)

${ }^{1} \mathrm{i}-\mathrm{lab}$, Key Laboratory of Multifunctional Nanomaterials and Smart Systems,

Suzhou Institute of Nano-Tech and Nano-Bionics (SINANO), Chinese Academy of Sciences (CAS), 398 Ruoshui Road, Suzhou, Jiangsu 215123, P. R. China

${ }^{2}$ Nano Science and Technology Institute, University of Science and Technology of China, 96 Jinzhai Road, Hefei, Anhui 230026, P. R. China

Full list of author information is available at the end of the article
}

including serpentine and accordion bellow shapes ${ }^{17-19}$. Metal-based devices take advantage of their electrical conductivity compared with their polymer-based counterparts. However, the brittleness and modulus mismatch between metals and soft substrates is a challenge that cannot be ignored in device design and fabrication ${ }^{20,21}$.

Strain isolation, a strategy to protect the active region by modifying the intrinsic rigidity contrast between metalbased devices and soft substrates, offers a possible solution to overcome the above problems ${ }^{22-25}$. Usually, delicate mechanical design is required to achieve reliable strain isolation, which includes assembling inorganic components on surface relief patterns rather than on flat surfaces ${ }^{26,27}$, embedding hard platforms within soft substrates ${ }^{22}$, and inserting a soft layer between devices and substrates ${ }^{25}$. However, there the use of strain isolation strategies in device manufacture is considerably complex. For example, strain isolation typically demands the use of transfer printing, in which active components in a designed layout are deposited on a silicon wafer and then transferred onto an elastomeric substrate in two separate steps ${ }^{28}$. Due to complexities in the preparation process, it is still a challenge to fabricate a thin

\section{(c) The Author(s) 2021}

(c) (i) Open Access This article is licensed under a Creative Commons Attribution 4.0 International License, which permits use, sharing, adaptation, distribution and reproduction cc) in any medium or format, as long as you give appropriate credit to the original author(s) and the source, provide a link to the Creative Commons license, and indicate if changes were made. The images or other third party material in this article are included in the article's Creative Commons license, unless indicated otherwise in a credit line to the material. If material is not included in the article's Creative Commons license and your intended use is not permitted by statutory regulation or exceeds the permitted use, you will need to obtain permission directly from the copyright holder. To view a copy of this license, visit http://creativecommons.org/licenses/by/4.0/. 
(submicrometer) metal-based device to satisfy the condition of conformal contact ${ }^{7,29}$.

Herein, inspired by the mechanism of strain isolation, we propose a facile in situ Joule heating method to directly fabricate stable epidermal electronics on PVA substrates. The intrinsic stiffness contrast in the polymer substrate obtained by Joule heating offers a simple yet robust mechanism without resorting to transfer printing. With an in situ Joule heating treatment, programmable changes in the crystallinity distribution can be achieved in the coplanar PVA substrate, resulting in a designed swelling rate and stiffness of the treated membrane. When the polymer substrate contact skin, the region without treatment absorbs moisture from the skin, and the membrane swells, resulting in a thinner substrate and perfect adapting to skin folds. To demonstrate the advantage of this method, metal-based surface electromyography (sEMG) sensors with low impedance, highly comfortable wearability, and good conformal adhesion properties were fabricated. In addition, human hand gesture classification based on the fabricated sEMG sensors was implemented, and a high classification accuracy of over $91.83 \%$ was achieved.

\section{Results and discussion \\ Properties of PVA membranes at different thermal treatment temperatures}

In this research, a nontoxic, easily processable, and biocompatible PVA membrane, was employed as the substrate. Previous reports indicated that the hydrogen bonds formed between PVA molecules could hinder crystallization of the membrane ${ }^{30}$; thus, a decrease in the swelling rate of PVA is associated with an increase in PVA crystallinity ${ }^{31}$. The crystallization of PVA can be enhanced by removing water through thermal treatment. Fig. 1a shows a schematic diagram of the crystallization process during the thermal treatment of PVA. At high temperature, the gradual loss of water in the PVA membrane leads to a decrease in the number of hydrogen bonds, and PVA crystallization occurs during the cooling process. By precise control of the thermal treatment temperature, different degrees of PVA crystallinity can be realized, as indicated by the XRD patterns in Fig. $1 \mathrm{~b}$. The gradual decrease in the full width at half maximum (FWHM) of the diffraction peak at $19.8^{\circ}$ with increasing annealing temperature is an indicator of the improvement in crystallinity. In addition, the intensity of the absorption peak at $1141 \mathrm{~cm}^{-1}$, which is attributed to the asymmetric stretching vibration of the $\mathrm{C}-\mathrm{C}$ bond ${ }^{31,32}$, significantly increases with increasing annealing temperature, as illustrated in Fig. 1c. These results confirm that the degree of crystallinity of the PVA membrane can be precisely controlled by tuning the thermal treatment temperature, thus further influencing the swelling rate of the PVA membrane when exposed to moisture, as shown in Fig. 1d. When the treatment temperature is lower than $100^{\circ} \mathrm{C}$, the PVA substrate completely dissolves in water. As the temperature increases, the swelling rate of the substrate decreases, and almost no swelling occurs when the annealing temperature reaches $170^{\circ} \mathrm{C}$. As a result, the thickness of the substrate after swelling decreases with decreasing annealing temperature, as indicated by the red curve in Fig. 1d. In addition, as illustrated in Fig. 1e and Fig. S1, the modulus of the PVA membrane increases rapidly when the temperature reaches $100^{\circ} \mathrm{C}$ and becomes saturated thereafter. All these results indicate that it is possible to control the thickness and Young's modulus of PVA membranes by modulating the thermal treatment temperature. Figure if shows an example showing that after annealing at $130^{\circ} \mathrm{C}$ for $30 \mathrm{~min}$, the PVA membrane is able to conformably adapt to skin folds after absorbing moisture from the epidermis and environment.

\section{Fabrication procedure of the metal layer on a PVA membrane}

Based on this, we fabricated a patterned metal layer on a PVA substrate and optimized the stress and modulus mismatch between the metal and PVA membrane through precise Joule heating treatment. As demonstrated in Fig. 2a, layers of polymethyl methacrylate (PMMA) and PVA were initially spin-coated on a glass substrate and cured at $130{ }^{\circ} \mathrm{C}$ for $30 \mathrm{~min}$. Then, chromium $(10 \mathrm{~nm})$ and gold $(100 \mathrm{~nm})$ were deposited on the substrate as a metal layer, which was patterned by electron beam evaporation through a hard mask, followed by spin-coating another layer of PVA on top as a protection layer. Afterward, a Joule heating process was carried out only on the PVA/metal layer/PVA area with a custom system, consisting of heating probes, a microscope, and a DC power supply, as shown in Fig. S2. After thermal treatment, the PVA membranesandwiched metal layer exhibits a high modulus and loses its swelling property, while other areas without annealing can still absorb moisture and swell when exposed to a moisture-rich environment.

Figure $2 \mathrm{~b}$ shows the as-prepared patterned metal layer (metal line, $300 \mu \mathrm{m}$ ) on the same PVA membrane without thermal treatment. Clear cracks appear on the metal, and the metal line is destroyed (Fig. 2d). In contrast, the metal layer on the PVA membrane after annealing remains intact, and the regions near the metal show good swelling properties and can adhere conformably to arbitrary surfaces (Fig. 2c and e). After thermal treatment, the stiffness of the PVA substrate increased, as reflected by its measured hardness increase (Fig. 2f). These results are also consistent with the modulus change of PVA with thermal treatment at $130^{\circ} \mathrm{C}$ and $170^{\circ} \mathrm{C}$ (Fig. 2g). In addition, thermal treatment of metal areas can decrease their strain. This can 


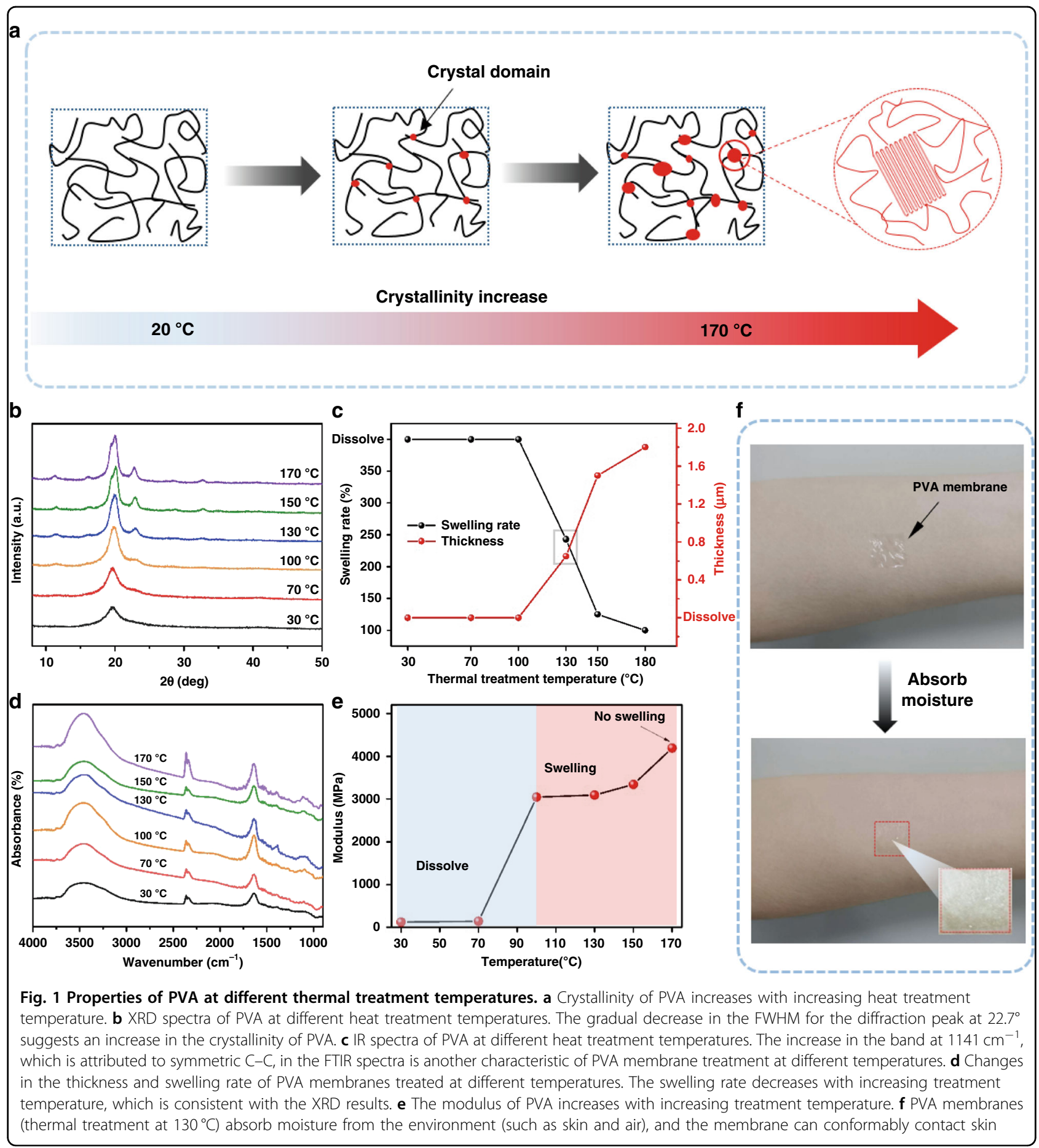

be explained by Hooke's law:

$$
\Delta l=\frac{F_{N} l}{E A}
$$

where $\Delta l$ is the elongation of the solid material, $l$ is the original length of the material, $F_{N}$ is the force applied to the material, $E$ is the modulus of the material and $A$ is the crosssectional area of the material. As illustrated in Fig. $2 \mathrm{~h}$, without thermal treatment, the thickness of the PVA substrate decreases. Therefore, the metal layer in the active region cannot be effectively protected by the PVA substrate, and obvious cracks appear. However, when the substrate is annealed with the in situ Joule heating treatment, the PVA 
a Spin coating
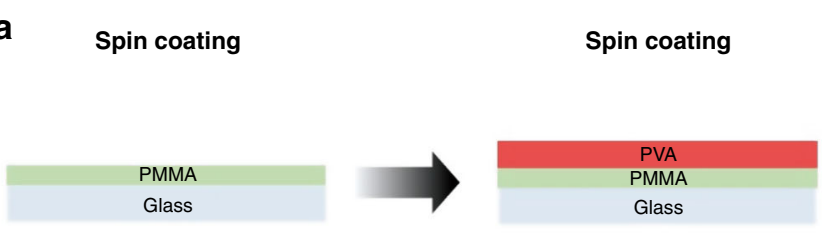

Glass

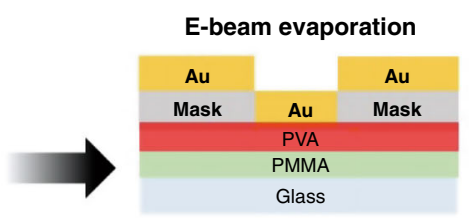

Release in acetone
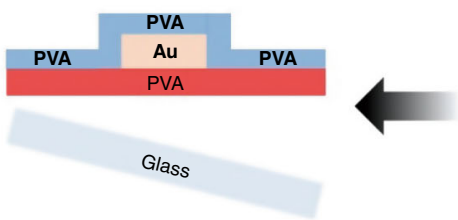

b
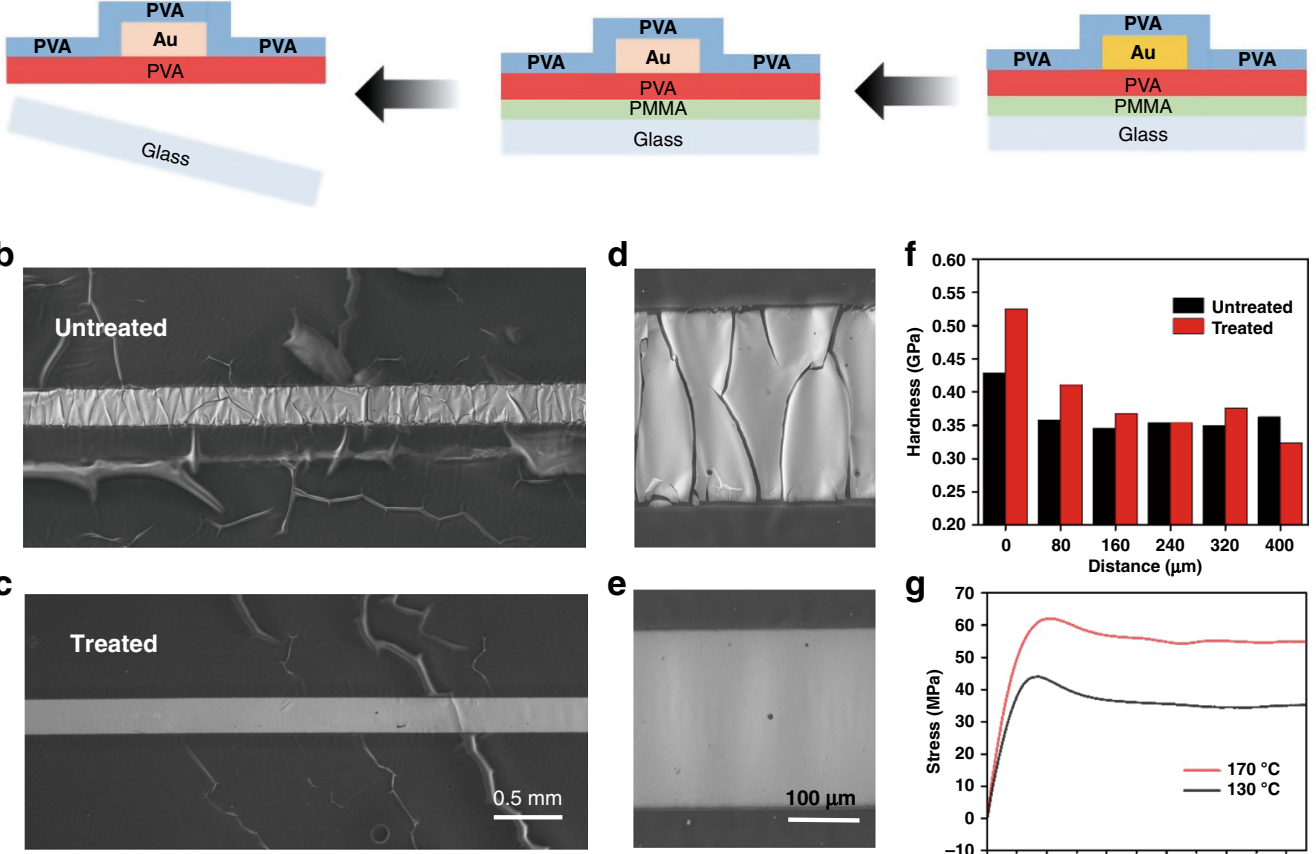

e

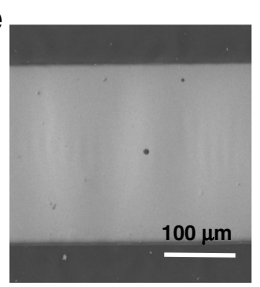

g

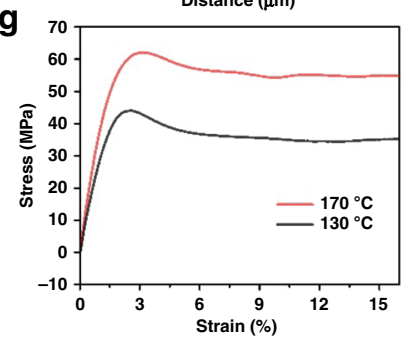

h.

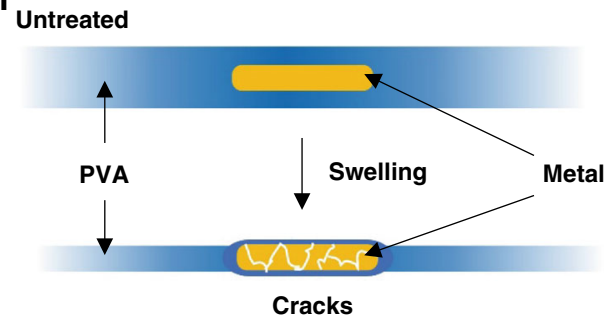

i Treated

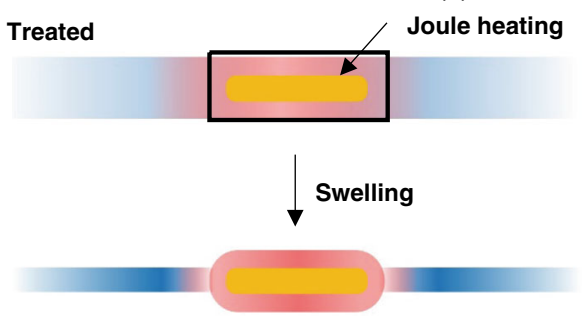

No cracks

Fig. 2 Schematic diagram showing the fabrication procedure of a metal layer on a PVA membrane. a Fabrication process of patterning metal layers on a PVA membrane. $\mathbf{b}$ The PVA membrane swells when exposed to a moisture-rich environment, and cracks appear for the metal line without any heat treatment. $\mathbf{c}$ Negligible deformation of the metal line occurs when it is subjected to heat treatment. SEM images of $\mathbf{d}$ untreated and $\mathbf{e}$ heattreated metal lines. The influence of heat treatment on the hardness of the PVA substrate is shown in $\mathbf{f}$, where a distance of 0 is the edge of the metal line. $\mathbf{g}$ Modulus of the PVA membrane with thermal treatment temperatures of $130^{\circ} \mathrm{C}$ and $170^{\circ} \mathrm{C}$. It is verified that the modulus of PVA surrounding the Joule heating metal layer is enhanced but changes little in other areas. Schematic diagrams of the cross-section of a PVA membrane with a metal layer. After absorbing moisture, $\mathbf{h}$ cracks appear on the metal layer without thermal treatment, but $\mathbf{i}$ the metal layer with thermal treatment remains complete and protected by the lack of swelling of the PVA membrane

substrate in the active region does not significantly swell when exposed to a moisture-rich environment, and no cracks appear on the metal layer (Fig. 2i). The decreased thickness of the untreated region and the increased modulus in the treated region both contribute to the decreased strain in the thermally treated area. Therefore, the thermal treatment area can be protected by strain isolation, and self-adhesion of the substrate is achieved after swelling. 

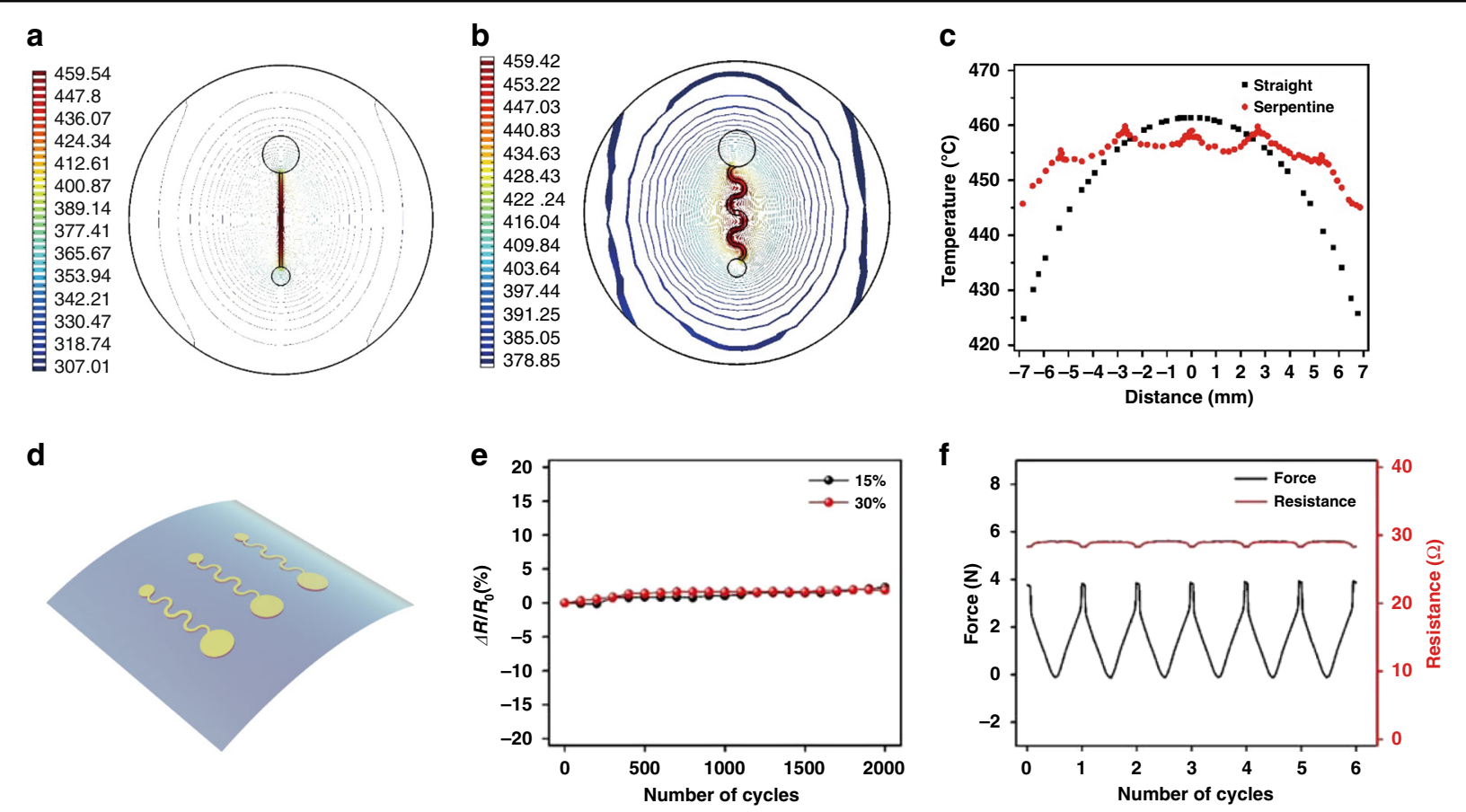

Fig. 3 Structural design and mechanical performance of the sEMG sensors. $\mathbf{a}$, $\mathbf{b}$ Heat distribution isotherm diagrams for sEMG sensors with a straight line and a serpentine metal line, respectively. A more uniform temperature distribution can be achieved for the serpentine metal line based on simulations. $\mathbf{c}$ The difference in the temperature can reach $36^{\circ} \mathrm{C}$ for the sensor with a straight design, which is $\sim 20^{\circ} \mathrm{C}$ higher than that of the serpentine metal sensor. $\mathbf{d}$ Schematic illustration of an sEMG sensor with a PVA substrate. e Resistance change of the sEMG sensors under 15 and $30 \%$ stretching on a skin replica for 2000 cycles. $\mathbf{f}$ A resistance change within $2 \Omega$ is measured during each stretching cycle (30\%)

\section{Structural design and mechanical performance of the sEMG sensors}

Based on the above results, sEMG sensors with long-term reliability can be fabricated with the proposed method. In addition, to improve device performance, we applied the finite element method (FEM) to simulate the temperature field distribution and optimize the structural design of the sensors. The temperature distribution of a straight-line sensor is shown in Fig. 3a, where the highest temperature appears in the center and decreases rapidly when moving away from the center. A significant temperature difference of $\sim 35.6^{\circ} \mathrm{C}$ between the center and margin areas is measured for the straight-line structure (black curve in Fig. 3c), which leads to a large difference in mechanical strength among these areas. In addition, a large temperature difference affects the swelling rate of the substrate and influences its adhesion to skin, thus degrading and damaging the metal layer due to the bulging of the PVA substrate (Fig. S3). In comparison, the temperature difference of the metal layer between the center and the ends for the serpentine structure design is only $14.7^{\circ} \mathrm{C}$, which is much lower than that of the straight structure (Fig. $3 \mathrm{~b}$ and c). This observation was further verified by experiments, where a temperature variation of $15.8{ }^{\circ} \mathrm{C}$ was obtained, as shown in Fig. S4.

Based on the simulation results, we fabricated a serpentine-shaped sEMG sensor on a PVA substrate
(Fig. 3d). A photograph and the structure design of the prepared sensor are shown in Figs. S5 and S6, respectively. To investigate the stretchability of the sensor, we fixed the device on a Mark-10 digital force gauge and measured its resistance fluctuation during stretching. When stretching the sensor to 15 or $30 \%$, the resistance of the device for both cases only changes within 3\% after 2000 cycles (Fig. 3e). In addition, during a single stretching cycle with a stretching rate up to $30 \%$ and a stretching force reaching $3.8 \mathrm{~N}$, the resistance change of this device is $2 \Omega$ (Fig. 3f). These results indicate that this sensor can endure the impact of people's daily actions; thus, stable sEMG signals can be obtained using this sensor.

\section{Electrical performance of the SEMG sensors}

Next, we placed the sEMG sensor on skin in a moisturerich environment and collected EMG signals (Fig. 4a). As expected, the PVA substrate absorbed moisture and became thinner allowing the sensor to conformally attach to the skin (Fig. 4b) due to swelling of the membrane. As illustrated in Fig. 4c, the sensor adapts well to skin folds, and no obvious cracks are observed.

Benefitting from their excellent mechanical stability, the sEMG sensors exhibit good electrical performance. First, impedance, which is an important factor that influences sEMG signals, was measured. Compared with commercial 
a

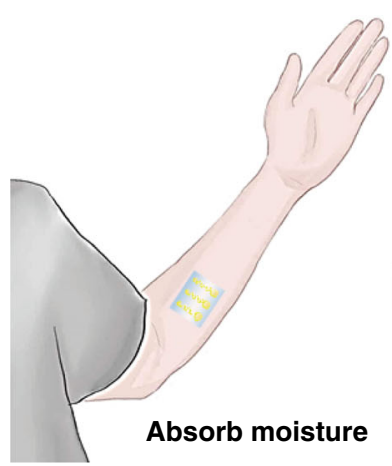

b
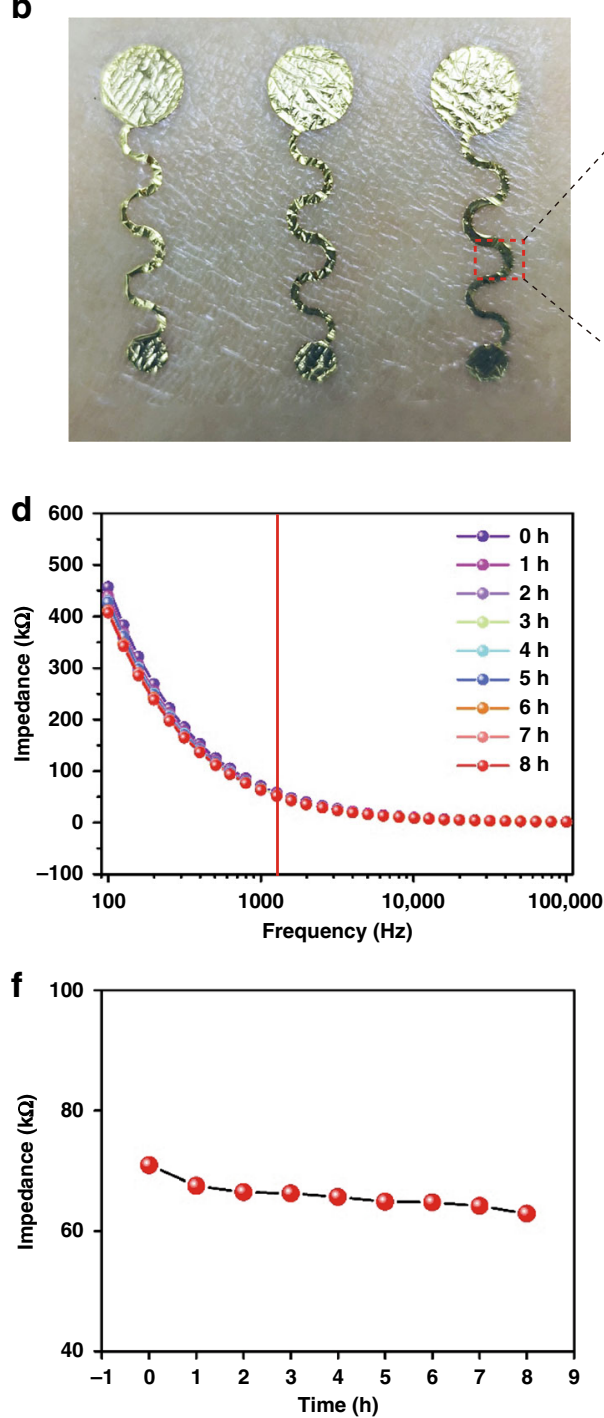

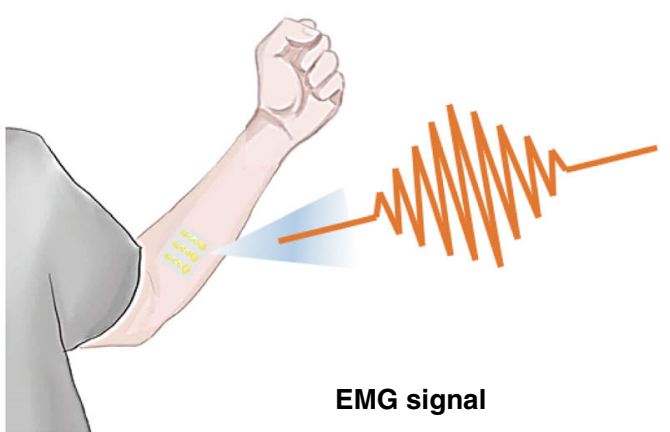

C
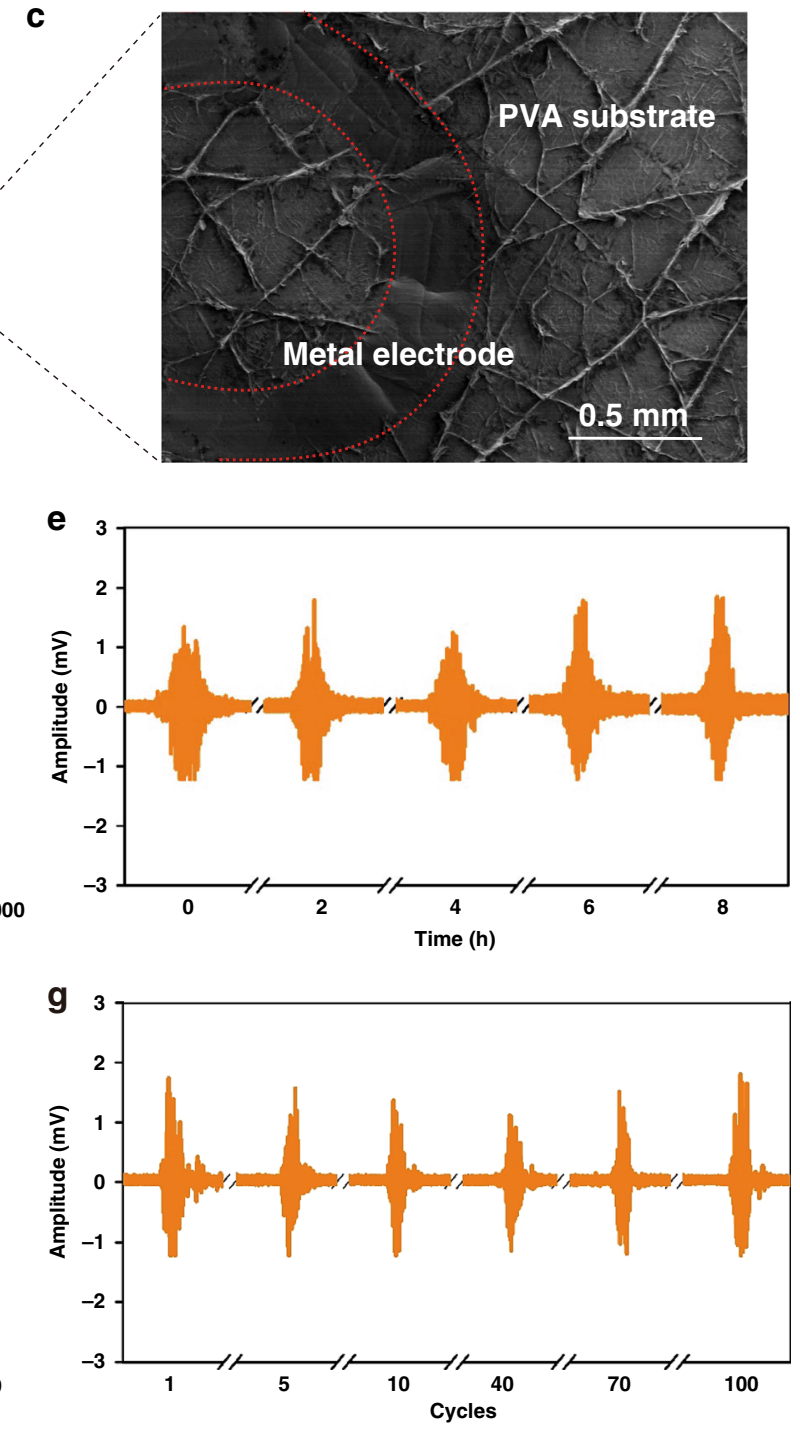

Fig. 4 Electrical performance of the sEMG sensors. a After absorbing moisture from the environment, the sensors can conformably fit skin folds and record SEMG signals. $\mathbf{b}$ Image of the sEMG sensors attached to human skin. c SEM image of a sensor that has been peeled off of skin. The area circled in red is the metal layer, which combines well with the substrate. $\mathbf{d}$ No significant increase in the impedance of the sensors is observed when attached to skin for $8 \mathrm{~h}$. e sEMG signals measured with the sensors. $\mathbf{f}$ No significant changes in the impedance of the sensors at $1 \mathrm{kHz}$ are observed. $\mathbf{g}$ sEMG signals for a series of muscle contractions followed by rest measured with the as-prepared sensors attached to the wrist 
gel sEMG sensors, the fabricated sEMG sensors exhibit better long-term stability ${ }^{33}$, which is of great importance in long-term monitoring ${ }^{29}$, despite a slight increase in impedance. As shown in Fig. $4 \mathrm{~d}-\mathrm{g}$, negligible changes in impedance are observed after $8 \mathrm{~h}$ of use. The decrease in impedance from 75 to $65 \mathrm{k} \Omega$ at a $1 \mathrm{kHz}$ frequency in the first hour can be attributed to the initial adaptation of the sensor to skin folds. During the $8 \mathrm{~h}$, the sensors were bent 100 times, and no significant degradation of the EMG signals is observed. These results further verify the stability of the device.

\section{Gesture classification of multichannel sEMG signals}

To verify the advantages of the sEMG sensors, we compared the recognition rate of captured signals between the prepared sEMG sensors and commercial sEMG sensors. Eight sensors were wrapped around a wrist at a sampling rate of $1000 \mathrm{~Hz}$, among which six were test sensors and the other two were a reference sensor and ground sensor. A volunteer was asked to repeatedly perform six defined gestures in sequence 100 times, and a total of 600 corresponding gesture signals were recorded.

Then, PyTorch software, including a manual segmentation module, a data preprocessing module, and a classifier module, was used to analyze and recognize gesture signals, as shown in Fig. 5a. The segmentation module starts by manually labeling the peaks of the sEMG wave and subsequently obtains 4500 values as segmented gesture data. Then, the above 4500 values are compressed into a $64 * 64 * 6$ matrix through the data processing module. After that, the classifier module inputs the matrix into DenseNet $100^{34}$. After 200 epochs of training, a classifier model and parameters based on sEMG gesture signals are obtained. Figure $5 \mathrm{~b}$ presents the sEMG signals of different hand gestures extracted through the six channels. The signal-to-noise ratio (SNR) of the sEMG signals during the test time is illustrated in Fig. S7. With increasing operation time, the SNR of the sEMG signals acquired by the commercial sensors decreases from 24.3 to 6.5. In contrast, the SNRs of the sEMG signals acquired by the prepared sensor are within the range of 19.8-23.4. As shown in Fig. 5c, the recognition rate slowly increases as training progresses for both the commercial and fabricated sensors in this experiment, and the recognition rate becomes saturated at a certain value. This result indicates that the model used in this research was convergent and that overfitting was avoided.

The accuracy for each individual test is presented in Fig. $5 \mathrm{~d}$. According to the statistical results, the average accuracy rates for the commercial and fabricated sensors were $82.33 \%$ and $91.83 \%$, respectively. The above results also demonstrate that in the case of long-term use, the fabricated sensors degrade more slowly than commercial sensors, resulting in a higher recognition rate.

\section{Conclusion}

In this work, strain isolation theory was applied to epidermal electronics, and a new method based on this theory was proposed to fabricate sEMG sensors on PVA membranes. With the metal devices undergoing a precise Joule heating process, the stiffness of the active area was enhanced, while the adhesion of the substrate was not affected. The optimal structure of the sEMG sensors was designed using the FEM. The sensors showed outstanding performance in terms of wearable comfort and stability compared with commercial gel sensors. Furthermore, to demonstrate the potential application of the sensors in HMIs, the sensors were shown to be able to recognize the sEMG signals of human gestures using machine learning algorithms with a high accuracy of $91.83 \%$. These results indicate that this method provides an idea to fabricate epidermal electronic devices in an efficient but robust way.

\section{Experimental section \\ Preparation of the PVA membrane}

A $12 \%$ PVA (PVA-124, Aladdin) solution was obtained by dissolving PVA-124 in deionized water and stirring for $30 \mathrm{~min}$ at $100^{\circ} \mathrm{C}$. PMMA (P141443, Aladdin) solution was obtained by dissolving PMMA powder in ethyl lactate (Aladdin) at a $30 \%$ weight ratio and stirring for $50 \mathrm{~min}$ at $150^{\circ} \mathrm{C}$. First, the PMMA solution $(2 \mathrm{ml})$ was coated on a 2 -inch glass substrate by spin coating $(3000 \mathrm{rpm}, 30 \mathrm{~s})$ and baked at $70{ }^{\circ} \mathrm{C}$ on a hot plate for $10 \mathrm{~min}$, which was used as a sacrificial layer. Then, a PVA layer $(2 \mathrm{ml})$ was coated on the PMMA layer by spin coating $(4000 \mathrm{rpm}, 30 \mathrm{~s})$ and baked at $130{ }^{\circ} \mathrm{C}$ on a hot plate for $30 \mathrm{~min}$.

\section{Material properties}

XRD was performed to investigate the crystallinity of PVA before and after heat treatment (Bruker D8). FTIR spectra were collected via a Thermo Nicolet iN 10 spectrometers with a laser operating at $77 \mathrm{~K}$ in a liquid nitrogen environment. Tensile tests were carried out using a universal material testing machine. A hardness test was carried out by a nanoindentation tester in continuous stiffness measurement mode. The distance between each test point was $80 \mu \mathrm{m}$, and the depth limit of every test point was $300 \mathrm{~nm}$.

\section{Structural design of the sEMG sensors}

The appropriate structure of the sEMG sensors was investigated by performing $\mathrm{FEM}$ and the $\mathrm{AC} / \mathrm{DC}$ module and heat transfer module were required to simulate electrical heat generation and heat transfer. Joule heating treatment models were established by drawing a geometric model of the sensor in simulation software and defining boundary conditions to simulate 


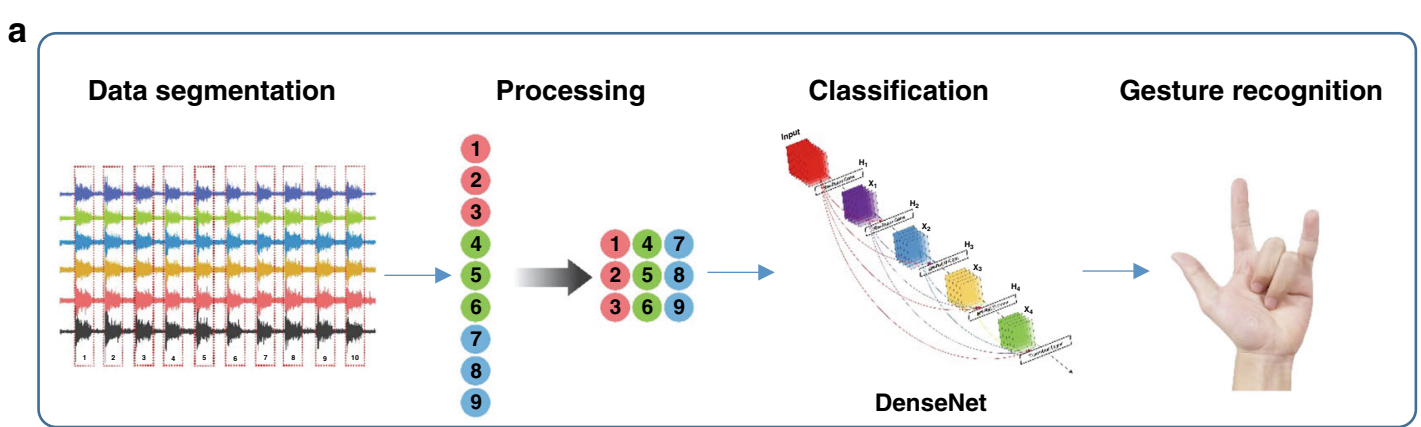

b
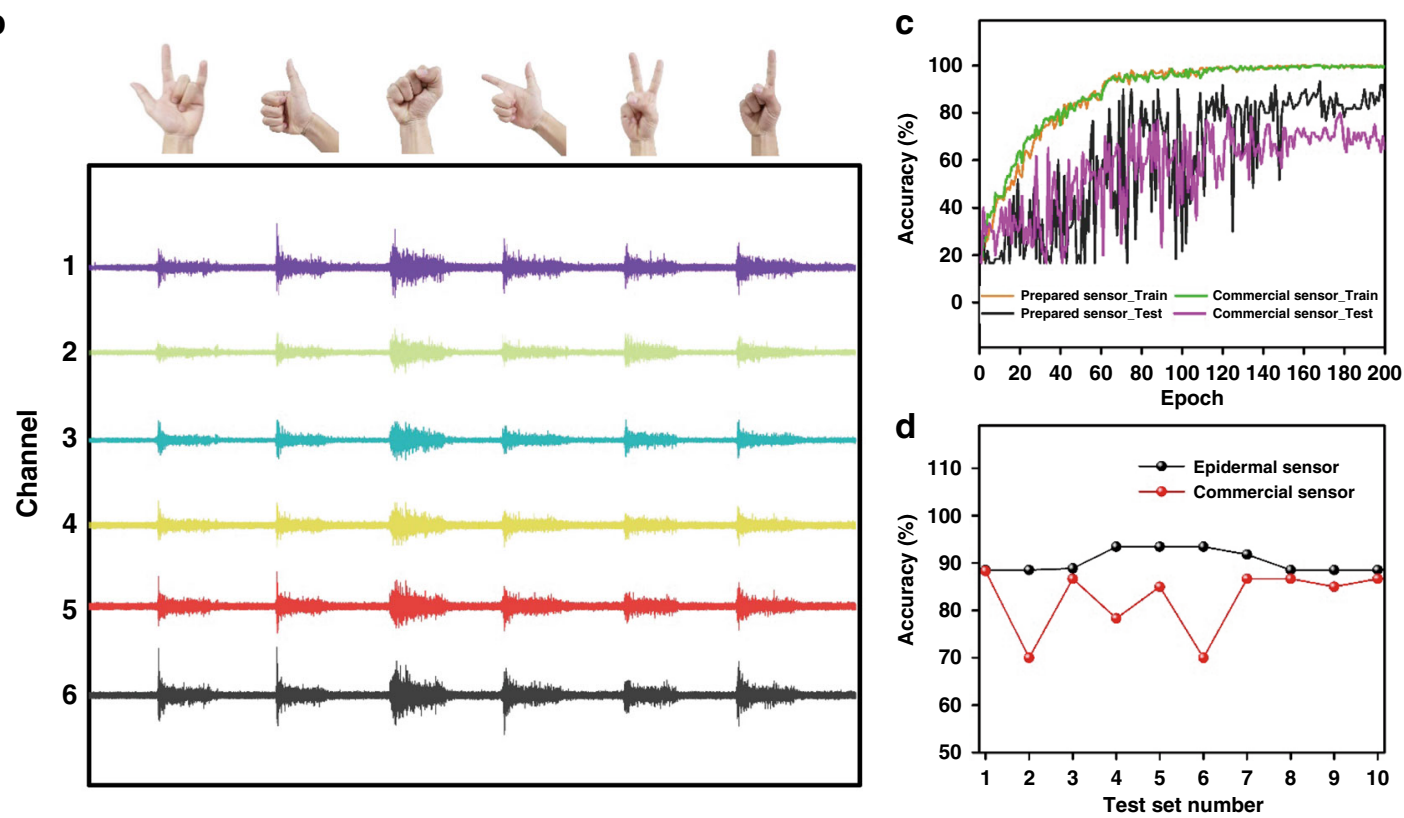

Fig. 5 Gesture classification of multichannel sEMG signals. a Structure of the program. The SEMG signal is segmented into ten parts, and one gesture is included in each part. Then, the data are processed into a $64^{*} 64$ matrix. Next, the matrix is input into DenseNet to classify the gestures. Finally, the gesture is recognized. $\mathbf{b}$ sEMG signals for six different gestures. c Training process of the data: the accuracy of the training increased as the number of epochs increased. The accuracy of the epidermal sensor was higher than that of the commercial gel sensor. $\mathbf{d}$ Recognition accuracy with different test sets. The accuracy of the prepared sensor was better than that of the commercial sensor while also being more stable

the real situation. The temperature distribution in the model can be more uniform by optimizing the size (line width, arc radius, etc.) of the model.

\section{Fabrication of the sEMG sensors}

A metal mask was laminated on the PVA membrane, and $\mathrm{Ti}$ and $\mathrm{Au}$ layers with thicknesses of $10 \mathrm{~nm}$ and $100 \mathrm{~nm}$ were deposited using E-beam evaporation (Ei$5 \mathrm{z}$, ULVAC). The deposition rate was set at $1.5 \AA$ per second. Then, another PVA layer was coated on the metal as a protective layer (spin-coating, $3000 \mathrm{rpm}$, $30 \mathrm{~s}$ ), followed by annealing at $130{ }^{\circ} \mathrm{C}$ for $20 \mathrm{~min}$. After that, a custom heating system was used to carry out the Joule heating process with an applied voltage of $8 \mathrm{~V}$ for 10 min. Finally, the PVA membrane with sEMG sensors was peeled off from the glass by dissolving the PMMA in acetone (immersed in acetone at $60^{\circ} \mathrm{C}$ for $20 \mathrm{~min}$ ).

\section{Characterization of the devices}

The temperature distribution test was carried out by a thermocouple thermometer (Kaipusen TES 1310) with the temperature probe fixed on the probe table. The temperature distribution of the metal line was monitored by moving the temperature probe to different places and testing the temperature.

The mechanical performance was investigated using a Mark-10 digital force gauge. The details are as follows: the sEMG sensor was placed on a skin replica made of PDMS (Dow Corning Sylgard 184), the sensor was stretched to the programmed length (15 and 30\%), and the impedance change of the sensor was investigated using a KEITHLEY 2602 instrument.

Skin/electrode interface impedance measurements were taken using an electrochemical workstation (Gamry Reference $600+$ ) with frequencies ranging from 100 to $10^{5} \mathrm{~Hz}$. 
The spacing between each electrode was maintained at $50 \mathrm{~mm}$. EMG signals were recorded by multiplex bioelectric data acquisition equipment (ZJE-II) at a sampling rate of $1000 \mathrm{~Hz}$. Commercial sensors with diameters of $\sim 50.5 \mathrm{~mm}$ were purchased from Liveyai Medical, which is a traditional $\mathrm{Ag} / \mathrm{AgCl}$ gel electrode for disposable use.

\section{Gesture recognition}

Eight sensors were wrapped around a wrist in a ring, six of which provided valid signals; the sampling frequency was $1000 \mathrm{~Hz}$. After one day of collection, the wearer repeated one of the six gestures, each movement lasted for approximately five seconds, and the same movement was repeated ten times before changing to the next gesture. Ultimately, 100 samples were collected for each gesture, reaching a total of 600 gesture samples. PyTorch was used to complete all experiments on a desktop computer with an NVIDIA 1060. As shown in Fig. 5a, to recognize the sEMG gesture signal, the program included a manual segmentation module, a data preprocessing module, and a classifier modul34e. The detailed steps of the segmentation module consist of first manually annotating the peaks of the sEMG wave and extracting the subsequent 4500 values as the segmented gesture data. The detailed steps of the data preprocessing module consist of compressing the above 4500 values into a $64 * 64$ matrix and then obtaining a $64 * 64 * 6$ matrix by stacking the six channels of data. The classifier module inputs the $64 * 64 * 6$ matrix into DenseNet100. After two hundred epochs of training, a classifier model and parameters based on the sEMG gesture signals are obtained. Considering the limited amount of experimental data, a tenfold cross-validation method was used to calculate the signal recognition rate 10 consecutive times.

\section{Acknowledgements}

The authors acknowledge funding support from the National Key R\&D Program of China $(2018$ YFB1304700) and the National Natural Science Foundation of China $(61574163,61801473)$.

\section{Author details}

${ }^{1}$ i-lab, Key Laboratory of Multifunctional Nanomaterials and Smart Systems, Suzhou Institute of Nano-Tech and Nano-Bionics (SINANO), Chinese Academy of Sciences (CAS), 398 Ruoshui Road, Suzhou, Jiangsu 215123, P. R. China. ${ }^{2}$ Nano Science and Technology Institute, University of Science and Technology of China, 96 Jinzhai Road, Hefei, Anhui 230026, P. R. China. ${ }^{3}$ School of Computer Science \& School of Cyberspace Science, XiangTan University, Yuhu District, Xiangtan, Hunan 411105, P. R. China

\section{Author contributions}

Z.W. conceived, designed, and performed experiments, analyzed data, and wrote the paper. Q.L. and Y.X. designed and performed experiments, analyzed data and wrote the paper. S.F., Y.S., S.W., X.Y. and Y.Z. reviewed and edited the paper. F.S. and T.L. reviewed the paper. T.Z. conceived and designed the experiments and wrote the paper.

\section{Conflict of interest}

The authors declare no competing interests.
Supplementary information The online version contains supplementary material available at https://doi.org/10.1038/s41378-021-00282-x.

Received: 18 January 2021 Revised: 20 April 2021 Accepted: 30 May 2021 Published online: 24 July 2021

\section{References}

1. Ko, H. C. et al. A emispherical electronic eye camera based on compressible silicon optoelectronics. Nature 454, 748-753 (2008).

2. Kim, D. H. et al. Epidermal electronics. Science 333, 838-843 (2011).

3. Nawrocki, R. A. et al. Self-adhesive and ultra-conformable, sub-300 nm dry thin-film electrodes for surface monitoring of biopotentials. Adv. Funct. Mater. 28, 1803279 (2018)

4. Wang, S. et al. Skin electronics from scalable fabrication of an intrinsically stretchable transistor array. Nature 555, 83-88 (2018)

5. Kwon, Y. T. et al. All-printed nanomembrane wireless bioelectronics using a biocompatible solderable graphene for multimodal human-machine interfaces. Nat. Commun. 11, 3450 (2020).

6. Xie, Z., Avila, R., Huang, Y. \& Rogers, J. A. Flexible and stretchable antennas for biointegrated electronics. Adv. Mater. 32, 1902767 (2020).

7. Ferrari, L. M. et al. Ultraconformable temporary tattoo electrodes for electrophysiology. Adv. Sci. 5, 1700771 (2018).

8. Jeong, J.-W. et al. Materials and optimized designs for human-machine interfaces via epidermal electronics. Adv. Mater. 25, 6839-6846 (2013).

9. Lai, S. et al. Ultra-conformable organic field-effect transistors and circuits for epidermal electronic applications. Org. Electron. 46, 60-67 (2017).

10. Koo, J. H. et al. Wearable electrocardiogram monitor using carbon nanotube electronics and color-tunable organic light-emitting diodes. ACS Nano $\mathbf{1 1}$ 10032--10041 (2017)

11. Yang, Y. et al. Facile fabrication of stretchable Ag nanowire/polyurethane electrodes using high intensity pulsed light. Nano Res. 9, 401-414 (2016).

12. Guo, W. et al. Matrix-independent highly conductive composites for electrodes and interconnects in stretchable electronics. ACS Appl. Mater. Interfaces $\mathbf{1 1}$ 8567-8575 (2019).

13. Kabiri Ameri, S. et al. Graphene electronic tattoo sensors. ACS Nano $\mathbf{1 1}$ 7634-7641 (2017)

14. Lim, S. et al. Transparent and stretchable interactive human machine interface based on patterned graphene heterostructures. Adv. Funct. Mater. 25, 375-383 (2015).

15. Choi, S. et al. Highly conductive, stretchable and biocompatible Ag-Au core-sheath nanowire composite for wearable and implantable bioelectronics. Nat. Nanotechnol. 13, 1048-1056 (2018)

16. Yang, $X$. et al. Ultrathin, stretchable, and breathable epidermal electronics based on a facile bubble blowing method. Adv. Electron. Mater. 6, 2000306 (2020).

17. $\mathrm{Xu}, \mathrm{S}$. et al. Stretchable batteries with self-similar serpentine interconnects and integrated wireless recharging systems. Nat. Commun. 4, 1543 (2013).

18. Jang, K. I. et al. Soft network composite materials with deterministic and bioinspired designs. Nat. Commun. 6, 6566 (2015).

19. Rogers, J. A., Someya, T. \& Huang, Y. Materials and mechanics for stretchable electronics. Science 327, 1603-1607 (2010).

20. Kim, D. H. et al. Ultrathin silicon circuits with strain-isolation layers and mesh layouts for high-performance electronics on fabric, vinyl, leather, and paper. Adv. Mater. 21, 3703-3707 (2009).

21. Cao, Y. et al. Direct fabrication of stretchable electronics on a polymer substrate with process-integrated programmable rigidity. Adv. Funct. Mater. 28, 1804604 (2018).

22. Romeo, A., Liu, Q., Suo, Z. \& Lacour, S. P. Elastomeric substrates with embedded stiff platforms for stretchable electronics. Appl. Phys. Lett. 102, 131904 (2013).

23. Xu, S. et al. Soft microfluidic assemblies of sensors, circuits, and radios for the skin. Science 344, 70-74 (2014).

24. $\mathrm{Wu}, \mathrm{J}$. et al. A strain-isolation design for stretchable electronics. Acta Mech. Sin. 26, 881-888 (2010).

25. Cheng, $\mathrm{H}$. et al. An analytical model of strain isolation for stretchable and flexible electronics. Appl. Phys. Lett. 98, 061902 (2011). 
26. Lee, J. et al. Stretchable semiconductor technologies with high areal coverages and strain-limiting behavior: demonstration in highefficiency dual-junction GalnP/GaAs photovoltaics. Small $\mathbf{8}$, 1851-1856 (2012)

27. Lee, J. et al. Stretchable GaAs photovoltaics with designs that enable high areal coverage. Adv. Mater. 23, 986-991 (2011).

28. Nam, J. et al. Transfer printed flexible and stretchable thin film solar cells using a water-soluble sacrificial layer. Adv. Energy Mater. 6, 1601269 (2016).

29. Miyamoto, A. et al. Inflammation-free, gas-permeable, lightweight, stretchable on-skin electronics with nanomeshes. Nat. Nanotechnol. 12 907-913 (2017).
30. Peppas, N. A. \& Merrill, E. W. Development of semicrystalline poly(vinyl alcohol) hydrogels for biomedical applications. J. Biomed. Mater. Res. 11 423-434 (1977).

31. Wong, K. K. H., Zinke-Allmang, M. \& Wan, W. Effect of annealing on aqueous stability and elastic modulus of electrospun poly(vinyl alcohol) fibers. J. Mater. Sci. 45, 2456-2465 (2010).

32. Peppas, N. A. Infrared spectroscopy of semicrystalline poly(vinyl alcohol) networks. Makromol. Chem. 178, 595-601 (1977).

33. Meng, Y., Li, Z. B., Chen, X. \& Chen, J. P. A flexible dry micro-dome electrode for ECG monitoring. Microsyst. Technol. 21, 1241-1248 (2015).

34. Huang, G, Liu, Z, Pleiss, G, Maaten, LVD \& Weinberger, K Convolutional networks with dense connectivity. IEEE Trans. Pattern Anal. Mach. Intell. 1-1 (2019). 